School of Finance

University of St.Gallen

\title{
Price DyNAMICS IN ElectRICITY MARKETS
}

FLORENTINA PARASCHIV

WORKING PAPERS ON FINANCE NO. 2013/14

INSTITUTE OF OPERATIONS RESEARCH AND COMPUTATIONAL FINANCE (IOR/CF - HSG)

JULY 2013 


\title{
Price dynamics in electricity markets
}

Florentina Paraschiv

\begin{abstract}
With the liberalization of global power markets, modeling of exchange traded electricity contracts has attracted significantly the attention of both academic and industry. In this paper we offer an overview of the most common deseasonalization techniques and modeling approaches in the literature. We extract the deterministic component of EEX Phelix hourly electricity prices and we discuss different financial and time series models for their stochastic component. Additionally we apply Extreme Value Theory (EVT) to investigate the tails of the price changes distribution. Generally our results suggest EVT to be of interest to both risk managers and portfolio managers in the highly volatile electricity markets.
\end{abstract}

\section{Introduction}

Finding realistic models to describe electricity prices is essential for the valuation of power grids, for the risk managers in the estimation of risk measures as well as for portfolio managers to determine worst-case scenarios in very turbulent markets.

Electricity prices pose a particular challenge for researchers, given their main characteristics: seasonalities, mean reversion, extremely large price movements as well as negative prices. Seasonality represents the deterministic component of the prices. A successful modeling approach is based on a rigorous deseasonalization technique. Therefore the seasonal components of electricity prices are discussed here in detail and we further offer a review of the main procedures used in the literature to deseasonalize them.

Beside the deterministic component, electricity prices have also a stochastic component, given by the inefficient storing capacities for electricity or by the intense use of renewable energy over the last years for power generation. Thus, the pro-

Florentina Paraschiv

Institute for Operations Research and Computational Finance, University of St. Gallen, Bodanstrasse 6, CH-9000, St. Gallen, Switzerland, e-mail: florentina.paraschiv@unisg.ch 
duction has to follow the more or less inelastic demand and traders of electricity with physical delivery are forced to balance their accounts in every single hour, independently of the actual offers. This leads to extreme fluctuations in electricity prices, which make them difficult to forecast. The most frequently applied models for the stochastic component of electricity prices are financial and time-series models. In this paper we offer a comparative view of three popular financial models: Brownian motion, Ornstein-Uhlenbeck process and the well-known Pilipovic model. From the time-series models we selected for discussion the most popular ones: ARMA (Autoregressive Moving Average) models as well as GARCH (Generalized Autoregressive Conditional Heteroscedasticity) models. Given the extremely large price movements in electricity prices, we model extreme tail quantiles with Extreme Value Theory (EVT) applied to EEX Phelix hourly electricity prices. We show that this procedure describes more realistically extreme tail quantiles than the classical time-series models.

In the sections 2 and 3 we offer a description of the main characteristics of electricity prices and we discuss the main deseasonalization techniques. Section 4 classifies and discusses comparatively different modeling approaches for electricity prices. Section 5 shows an application of extreme value theory for modeling extremely large electricity price changes. Section 6 offers a summary of the paper.

\section{Characteristics of electricity prices}

Electricity markets have particularities which clearly distinguish them from other commodity markets. Given the lack of efficient storing opportunities for electricity, which prevents intertemporal smoothing of the demand by holding storages, extremely large price movements (spikes) as well as various cyclical patterns of behavior occur. Supply and demand determine market prices which have to correspond exactly at any location and at any time. Because of limited efficient storing capacities, the grid operators have difficulties to balance out hard-to-predict variations in power production and consumption in order to cover peak loads. The main characteristics of electricity prices are: seasonalities, mean reversion and negative prices.

\subsection{Seasonalities}

The seasonal behavior of electricity prices is one of the most complicated ones among all commodities. It is predominantly caused by the almost inelastic, at least in the short-term, demand for electricity, which by itself shows pronounced patterns caused by economic and business activities.

Electricity prices reveal three types of cyclical patterns: daily, weekly and yearly seasonality (see [3]). As emphasized in [27], the amount of electricity demanded de- 
pends mostly on the level of human activity, as well as on the weather and climate conditions. Essentially, two Off-Peaks can be observed: Off-Peak 1 and Off-Peak 2. Off-Peak 1 is represented by the first 7 hours of the day, when most people sleep and fewer businesses are operating. Off-Peak 2 are the last four hours of the day, when most human and business activities have ceased. In-between, during the Peak hours (between 8 a.m. and 8 p.m.), electricity demand increases drastically, an intensification that is linked to people getting ready for the day ahead and to the start of the business activities (see [5]). Figure 5 summarizes the autocorrelation function for the baseload hours (prices for the 24 hours of the day) versus the Off-Peak and Peak hours. On average, electricity prices are relatively constant during the working week (holidays have to considered separately), whereas at week-ends and during holidays electricity prices fall. In addition to this, the hourly pattern differs depending on the season. While in winter we can observe two peaks (at noon and one evening peak, at 7 p.m.), in summer we typically observe only one peak at noon (see figure 1).

\subsection{Mean-reversion}

In the short-term electricity prices are characterized by jumps or spikes. However, in the long-run they revert to the mean-reverting level (MRL) (see [6]). The longterm price level is characterized by the marginal costs of production. These can be constant, periodic or periodic with trend. This argument refers to the theory of perfect competition, i.e. if demand for electricity is high, production capacities with high marginal costs are implemented, whereas if demand is low, production capacities with low marginal costs are used and consequently prices fall. The alignment from capacities with lowest to ones with highest marginal costs is depicted by the so-called merit order. This notion implies that there is more than one constant mean-reversion level, depending on the time of the day, of the week, and of the year (see previous section). Hence, the concept of mean reversion implies that electricity prices return to their respective usual level (see [11]).

\subsection{Negative prices}

The limited storing capacities and the limited load change flexibility caused the negative electricity prices at EEX. From an economic perspective, negative prices can be rational, e.g., if the costs to shut down and ramp up a power plant unit exceed the loss for accepting negative prices (see [16]). Since September 1st 2008, negative price bids have been allowed at the German power exchange EEX as the first energy exchange in Europe. In our analysis we will refer to the German power exchange EEX Phelix hourly electricity prices between September 2008 and December 2011. Historical spot market data over the investigated period shows a total amount of about 100 hours with negative prices. Mostly, they occur in the night and morning 
hours (23:00 to 08:00) as displayed in figure 6. Furthermore, they occur with higher frequency on Mondays and Sundays (figure 7). As shown in the histogram in figure 8 , the absolute frequency of the prices presents clusters of 2 EUR/MWh.

\section{Deseasonalization techniques}

The electricity prices are explained by two fundamental components: a deterministic component represented by the typical seasonality pattern and the price uncertainty as stochastic component of the prices. The load, as one main driver of electricity prices shows some noticeable patterns, such as the peak at midday in summer days. The electricity prices follow more or less typical seasonality patterns, which are described in the literature by deterministic functions. However, as discussed in [16], beside the deterministic impact factors, electricity spot prices are also influenced by uncertain parameters like power plant outages and fluctuant renewable electricity generation. These uncertainties are drivers of the stochastic component of the spot prices. The current section offers an overview of the deseasonalisation techniques applied in the literature for electricity prices, while the next section will classify modeling approaches for the stochastic component.

\subsection{Preliminaries}

In order to incorporate the seasonal feature while taking the property of meanreversion into account, the spot price can be expressed as a combination of these two components:

$$
P_{t}=f_{t}+\sum_{i=1}^{n} X_{i, t}
$$

where $f_{t}$ is the deterministic component seasonality and $X_{t}$ is the stochastic part. There is a discussion in the literature whether level or logarithmic prices should be modeled. Accordingly to [31] or [15] the drawback of deseasonalizing day-ahead spot prices is that the residuals of $P_{t}-f_{t}$ can become negative, which prevents the use of logarithms on the deseasonalized spot prices. In addition, the authors mention as well that the advantage of using the spot prices instead of their logarithms is that on average they yield better parameter estimates. In the current paper we will look at the level of the prices.

Demand and supply of electricity show seasonal fluctuations, which traduce into the seasonal behavior of spot electricity prices. Figure 1 depicts the average price per month (January and July) and type of day (week-day and weekend day/holiday, respectively). This chart confirms the seasonality pattern of the electricity prices. Over the $24 \mathrm{~h}$ time period, prices move in a distinct hourly pattern, which follows the demand for electricity. As we have already mentioned, prices start increasing when 
people get ready for the day (around 6-7 a.m.) and decrease after 8 p.m., when business activities are over. We observe differences in the prices between winter and summer time as well as between workdays and weekend days (see [11]). Often researchers look distinctively at different hours within a day, given their distinctive patterns: they distinguish between baseload and Peak hours and even more: between Off-Peak I and Off-Peak II hours.

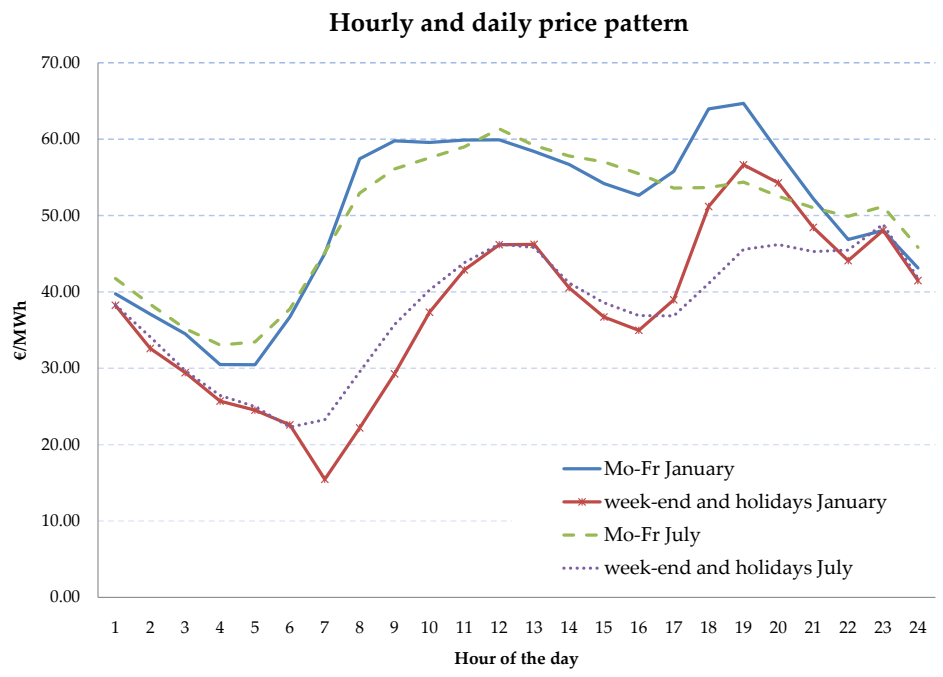

Fig. 1 Hourly and daily day-ahead price patterns for EEX Phelix

\subsection{Overview of deseasonalization techniques}

The aim of deseasonalization techniques is to reduce the predictable pattern of electricity prices, in order to delimitate the stochastic component of the prices. We firstly remove the long-term trend from the hourly electricity prices:

$$
f_{t}^{\text {trend }}=a^{\text {trend }}+b^{\text {trend }} t
$$

The constant term $a$ of the equation may be interpreted as the fix costs of power production. The term $t$ represents the "long-run linear trend in the total production cost", which is related to macroeconomic variables like inflation and, hence, depicts a positive trend. 
Secondly, the seasonality of de-trended prices should be removed. Based on the discussion in [11], we give an overview of the several suggestions in the literature to describe the daily, weekly and annual cycles of electricity prices. There are many deseasonalizing techniques in the literature. Thus, [17] implement piecewise constant functions; [8], [25] and [32] adopt sinusoidal functions; [19] use a combination of both.

\subsubsection{Yearly seasonality}

The yearly seasonality can be modeled with the classical trigonometric functions:

$$
f_{t}^{\text {season }}=a+b_{1, t} \cos \left(\frac{2 \pi}{8760} \cdot(t-\tau)\right)+b_{2, t} \sin \left(\frac{2 \pi}{8760} \cdot(t-\tau)\right)
$$

The parameter $\tau$ defines the phase shift, i.e., the starting point of a seasonal oscillation, and 8760 is the number of hours in one year. The use of trigonometric functions to define the yearly season is a common method in the literature. However, this method alone does not deliver satisfactory results (see [16]). The use of only trigonometric functions for the EEX prices is indeed not suitable, as they do not show a strong seasonality over the year - after all, some winters have almost spring-like temperatures, and vice versa. In order to make the explanatory power of the trigonometric functions stronger, other variables need to be added, e.g. time (see [31]).

Another method suggested by [16] is the use of the monthly average prices $\bar{P}_{m^{\prime}}$ as a seasonality factor over the year. The seasonality over the year in this case defines the mean values of the hours of the respective months as a seasonality component:

$$
f_{t}^{\text {season }}=\sum_{m^{\prime}=1}^{12} \bar{P}_{m^{\prime}} * 1\left(m^{\prime} \mid m^{\prime}=m(t)\right) \text {. }
$$

\subsubsection{Weekly seasonality}

The weekly cycle can be established using several methods. The first one is called adjusted absolute sinus-function (aasf) (see [16]).

$$
f_{t}^{\text {weekly }}=\alpha+d\left|\sin \left(\frac{\pi * t}{168}-\varphi\right)\right|
$$

The phase shift parameter $\varphi$ is determined as the deviation from the point in which the weekly cycle reaches its minimum in the observation set. For this purpose, we calculate the mean values of the electricity prices for each day of the week. This calculation delivers the seventh hour of Sunday as the minimum price. Then we take the distance of all hours of the week from the respective Sunday's seventh hour (see [16]). An alternative to verify to what extent a (daily) seasonality exists within the 
week is to include dummy variables. For more information about this method, see [3] or [11].

\subsubsection{Daily cycle}

The daily cycle is defined as the mean over the 24 hours of the day and is then removed. Different daily cycles are determined for each season: winter, spring, summer and autumn. Thus, we take daily average prices for each weekday $d$ dependent on the season.

$$
f_{i, \text { season }}^{\text {daily }}=\frac{24}{T_{\text {season }}} \sum_{d=0}^{\left(\frac{T_{\text {season }}}{24}\right)-1} P_{i+24 d, \text { season }}
$$

where $d$ is one of the weekdays, $i$ is the hour of the day and $T$ is the maximum number of hours overall $d$ weekdays in one season (winter, spring, summer or autumn). There are two ways used in the literature to deseasonalize: either to split the seasonal decomposition into daily, weekly and yearly seasonality and to estimate them separately, or to consider them simultaneously (see [16]):

$$
\begin{aligned}
f_{t} & =a^{\text {trend }}+b^{\text {trend }} t+c \frac{24}{T_{\text {season }}} \sum_{d=0}^{\left(\frac{T_{\text {season }}}{24}\right)-1} P_{i+24 d, \text { season }}+d\left|\sin \left(\frac{\pi * t}{168}-\varphi\right)\right|+ \\
& +e \sum_{m^{\prime}=1}^{12} \bar{P}_{m^{\prime}} * 1\left(m^{\prime} \mid m^{\prime}=m(t)\right)
\end{aligned}
$$

\subsection{Application}

In this section we derive the seasonality shape for EEX Phelix hourly electricity prices quoted at the European Energy Exchange (EEX), between September 2008 and December 2011. The derivation of the deseasonalization shape follows the procedure described in [3]. In a first step, we identify the seasonal structure during a year with daily prices. In the second step, the patterns during a day are analyzed using hourly prices. Let us define two factors, the factor-to-year $(f 2 y)$ and the factorto-day $(f 2 d)$ (following the usual notation in [3]). By $f 2 y$ we denote the relative weight of an average daily price compared to the annual base of the corresponding year:

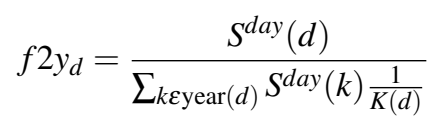


$S^{\text {day }}(d)$ is the daily spot price in the day $d$, which is the average price of the hourly electricity prices in that day. $K(d)$ denotes the number of days in the year when $S^{\text {day }}(d)$ is observed. The denominator is thus the annual base of the year in which $S(d)$ is observed.

To explain the $f 2 y$, we use a multiple regression model (similar to [3]):

$$
f 2 y_{d}=\alpha_{0}+\sum_{i=1}^{6} b_{i} D_{d i}+\sum_{i=1}^{12} c_{i} M_{d i}+\sum_{i=1}^{3} d_{i} C D D_{d i}+\sum_{i=1}^{3} e_{i} H D D_{d i}+\varepsilon
$$

- $f 2 y_{d}$ : Factor to year, daily-base-price/yearly-base-price

- $D_{d i}: 6$ daily dummy variables (for Mo-Sat)

- $M_{d i}: 12$ monthly dummy variables (for Feb-Dec); August will be subdivided in two parts, due to summer vacation

- $C D D_{d i}$ : Cooling degree days for 3 different German cities

- $H D D_{d i}$ : Heating degree days for 3 different German cities

where $C D D_{i} / H D D_{i}$ are estimated based on the temperature in Berlin, Hannover and Munich.

- Cooling Degree Days $(\mathrm{CDD})=\max \left(T-18.3^{\circ} \mathrm{C}, 0\right)$

- Heating Degree Days (HDD) $=\max \left(18.3^{\circ} \mathrm{C}-T, 0\right)$

We transform the series $f 2 y_{d}$ from daily to hourly, by considering the same factorto-year $f 2 y_{d}$ for each hour $t$ observed in the day $d$. In this way we construct hourly $f 2 y_{t}$ series, which later enter the shape $s_{t}$. The $f 2 d$, in contrast, indicates the weight of the price of a particular hour compared to the daily base price.

$$
f 2 d_{t}=\frac{S^{\text {hour }}(t)}{\sum_{k \varepsilon \text { day }(t)} S^{\text {hour }}(k) \frac{1}{24}}
$$

with $S^{\text {hour }}(t)$ being the hourly spot price at the hour $t$. We know that there are considerable differences both in the daily profiles of workdays, Saturdays and Sundays, but also between daily profiles during winter and summer season. Thus, following [3] we suggest to classify the days by weekdays and seasons and to choose the classification scheme presented in table 1. The workdays of each month are collected in one class. Saturdays and Sundays are treated separately. In order to obtain still enough observations per class, the profiles for Saturday and Sunday are held constant during three months.

Table 1 The table indicates the assignment of each day to one out of the twenty profile classes. The daily pattern is held constant for the workdays Monday to Friday within a month, and for Saturday and Sunday, respectively, within three months.

\begin{tabular}{l|cccccccccccc}
\hline \hline & J & F & M & A & M & J & J & A & S & O & N & D \\
\hline Week day & 1 & 2 & 3 & 4 & 5 & 6 & 7 & 8 & 9 & 10 & 11 & 12 \\
Sat & 13 & 13 & 14 & 14 & 14 & 15 & 15 & 15 & 16 & 16 & 16 & 13 \\
Sun & 17 & 17 & 18 & 18 & 18 & 19 & 19 & 19 & 20 & 20 & 20 & 17 \\
\hline \hline
\end{tabular}


The regression model for each class is built quite similarly to the one for the yearly seasonality. For each profile class $c=\{1, \ldots, 20\}$ defined in table 1 , a model of the following type is formulated:

$$
f 2 d_{t}=a_{o}^{c}+\sum_{i=1}^{23} b_{i}^{c} H_{t, i}+\varepsilon_{t} \quad \text { for all } t \varepsilon c .
$$

where $H_{i}=\{0, \ldots, 23\}$ represents dummy variables for the hours of one day.

The seasonality shape $s w_{t}$ can be calculated by $s w_{t}=f 2 y_{t} \cdot f 2 d_{t} . s w_{t}$ is the forecast of the relative hourly weights and it is additionally multiplied by the yearly average prices, in order to align the shape at the prices level. This yields the seasonality shape $s_{t}$ which is finally used to deseasonalize the electricity prices. Figure 2 shows the autocorrelation function of the hourly prices before and after deseasonalizing.
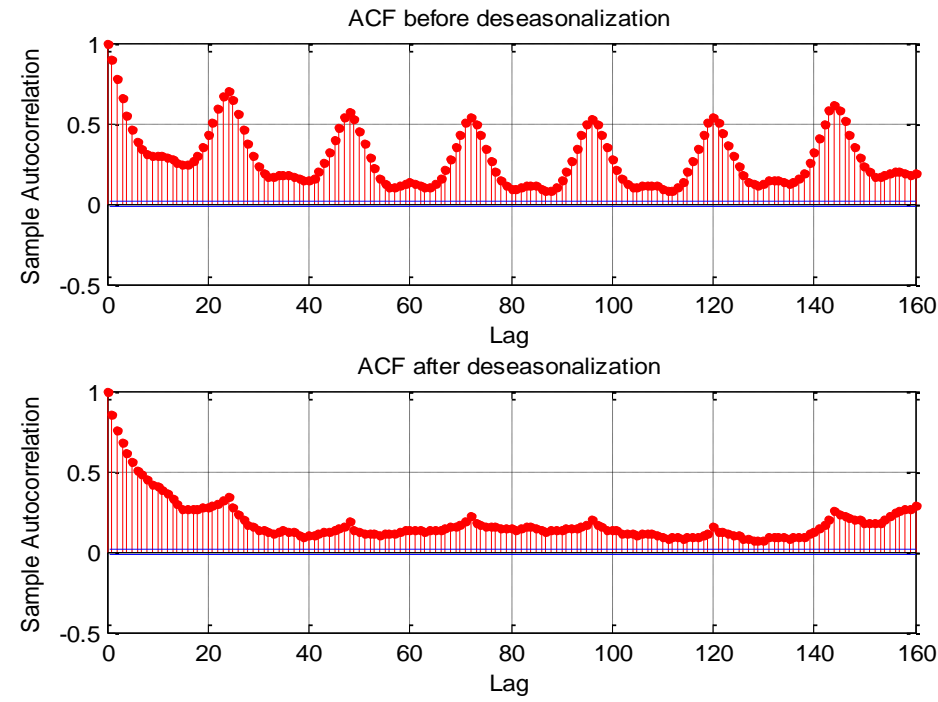

Fig. 2 Autocorrelation function before and after deseasonalization

The deseasonalized series is assumed to contain only the stochastic component of electricity prices, such as the volatility and randomly occurring jumps and peaks, which can be simulated via different stochastic processes, as described in the following section. 


\section{Modeling approaches for electricity prices}

There are many different theoretical methods that can be applied for electricity price simulations depending on the research question or planning tasks. Thus, the different methods cannot be directly compared with each other as each method has its strengths and its weaknesses. Accordingly to [30], these methods can be classified as:

- fundamental models

- game theoretic models

- financial mathematics models

- statistical and econometric time-series models

- technical analysis or expert system

\subsection{Fundamental models and game theoretic approaches}

Fundamental models consider modeling of the whole electricity system with all suppliers, whereas each single power plant or technology classes are described separately in the modeling approach. This type of models include a detailed analysis about the electricity demand as well as capacity use and maintenance hours of power plants. They are used to produce scenarios for electricity prices, which are further integrated for middle- to long-term planning tasks and price forecasts (see [16], [22], [29]).

Game theoretic approaches consider the strategic behavior of different market stakeholders [16]. These models simulate competitive electricity markets and analyze long-term equilibriums on the whole-sale market in general based on a Cournot-Nash framework (see [14], [18]). This type of models is used to test different market design options and to analyze the behavior of market participants (see discussion in [16]).

\subsection{Financial models}

Financial and time-series models are calibrated on historical hourly or daily prices and used for short-term price forecast in risk management. Financial mathematical models such as Geometric Brownian motions or mean-reversion processes are one of the most applied stochastic processes for electricity prices. They deal with the volatility of electricity prices and can be used for derivative pricing or real options in energy markets [13]. As an example for mean-reversion processes is the OrnsteinUhlenbeck process which, formulated for electricity price changes, reads:

$$
d X(t)=k_{1}\left(\mu_{1}-X(t)\right) d t+\sigma_{1} d W_{1}(t)
$$


The first term of the mean reversion process is the drift component. The parameter $k_{1}$ describes the speed of the reversion of the stochastic component of the electricity prices to their long-term mean $\mu_{1}$. The economic interpretation of this mean-reversion component is that stochastic price fluctuations around the mean and price peaks are only temporary, caused by e.g., power plant outages or capacity storages [16]. The second term, the stochastic component $d W_{1}(t)$ corresponds to the standard Brownian motion.

Another class of financial models is represented by two-factor models, which distinguish between the short- and long-term dynamics of the prices. Examples in this sense are the Pilipovic model or the model proposed by [26] who applied it to oil markets, and expanded by [19] and [20]. The Pilipovic model is well established in commodity markets and known as long-term/short-term model. The short-term deviations are explicitly modeled as the deviations from the long-term mean.

The two-factor [24] model under $\mathscr{P}$ is

$$
\begin{aligned}
d X_{t}^{U n d} & =k_{2}\left(L_{t}-X_{t}^{U n d}\right) d t+X_{t}^{U n d} \sigma_{2} d W_{2 t} \\
d L_{t} & =\mu_{2} L_{t} d t+L_{t} \sigma_{3} d W_{3 t} \\
d W_{2 t} d W_{3 t} & =0
\end{aligned}
$$

where

$\begin{array}{ll}X & \text { Spot price }\end{array}$

$X^{U n d} \quad$ Underlying spot price value

$L_{t} \quad$ Equilibrium price

$t \quad$ Time of observation

$k_{2} \quad$ Rate of price mean reversion

$\sigma_{2} \quad$ Volatility

$\mu_{2} \quad$ Drift of the long-term equilibrium price

$\sigma_{3} \quad$ Volatility of the long-term equilibrium price

$d W_{2} \quad$ Random stochastic variable defining the randomness in the spot prices

$d W_{3} \quad$ Random stochastic variable defining the randomness in equilibrium prices

The drift of the second factor reflects the expectations about available production capacities in the future, trend of demand or political or regulatory effects. The first factor, in contrast, models the differences between current value and a stochastic equilibrium level. This means that the level of mean-reversion is not constant, but it depends on the time of the day, of the week and of the year. These deviations reflect short-term effects that result, for example, from variations in weather conditions or intermittent supply shortages (see [3]).

To see the differences among the three popular financial models, we simulate electricity spot prices employing a Brownian motion, an Ornstein-Uhlenbeck process and the Pilipovic model. Firstly, we simulate the stochastic residuals using equations (12) and (13), and in a second step, to obtain spot prices, we add the sea- 
sonality shape, as derived in section 3.3. We simulate 1000 scenarios and we look comparatively at the probability distribution function of the simulated prices after 1 month, 6 months and 12 months horizon. The parameters used for our simulation are summarized in table 2 . Results are displayed in figure 3.
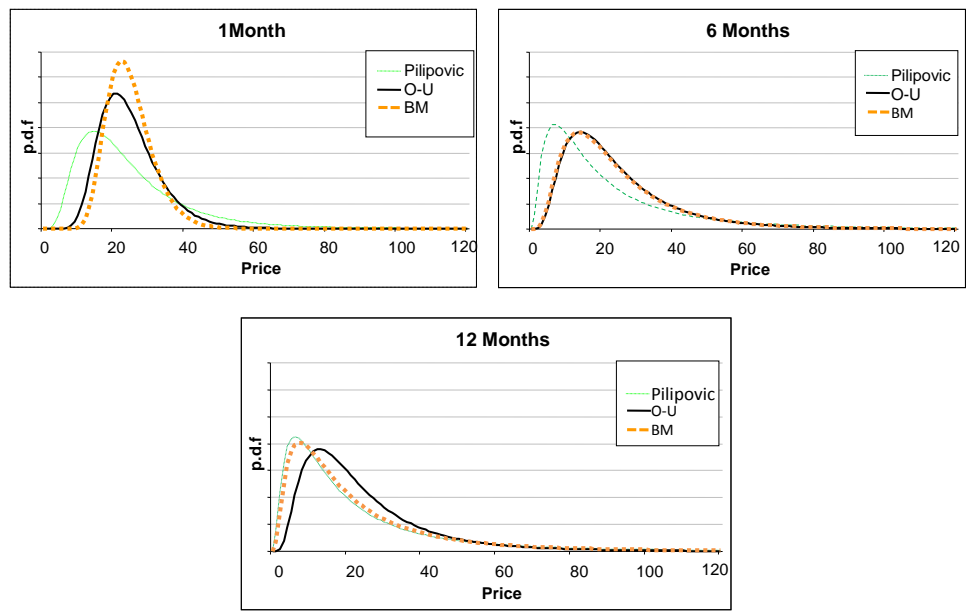

Fig. 3 Distributional information over scenarios generated with different financial models

Table 2 Simulation parameters per annum

\begin{tabular}{lc}
\hline \hline Geometrical Brownian motion \\
$\sigma_{0}$ & $0.9^{-1 / 2}$ \\
\hline \multicolumn{2}{l}{ Ornstein-Uhlenbeck process } \\
$k_{1}$ & $1.7^{-1}$ \\
$\mu_{1}$ & 0 \\
$\sigma_{1}$ & $0.74^{-1 / 2}$ \\
\hline Pilipovic & model \\
$k_{2}$ & $3^{-1}$ \\
$\sigma_{2}$ & $0.74^{-1 / 2}$ \\
$\sigma_{3}$ & $0.25^{-1 / 2}$ \\
$\mu_{2}$ & 0 \\
\hline \hline
\end{tabular}

We observe that the distribution of spot electricity prices simulated by the Pilipovic model is more skewed to the right than in the case of the prices obtained with the other two model versions. This is actually the more realistic distribution, given the extremely large price movements observed in electricity prices. 
The difference between the Pilipovic model and the other two (Brownian motion and Ornstein-Uhlenbeck models) is given by the fact that the former distinguishes between the short-term and the long-term dynamics of electricity prices. Furthermore, the log spot price is assumed to mean revert towards an equilibrium level, which itself is stochastic, while in the Ornstein-Uhlenbeck process the equilibrium level is assumed constant. All three models simulate the skewed shape of the distribution of electricity prices, with an increase in the planning horizon. However, the Pilipovic model offers more realistic short-term forecasts than the other two models.

\subsection{Time series models}

Due to their widespread use and their comprehensibility we discuss now an important class of time series models - the family of Autoregressive Moving Average (ARMA) models. This forecasting method is based on the assumption that data have an internal structure such as autocorrelation. ARMA processes enable the simulation of time dependencies within a time-series and consists of two parts, the autoregressive and the moving average part. The autoregressive component considers the lagged $p$-price values for computing the stochastic component of the electricity price $X_{t}$ in $t$. The moving average component takes the weighted mean of the last $q$ error terms into account. The calculation of the price in $t$ depends at least on a new error term $\varepsilon_{t}$, which can be, e.g., normally or Laplace distributed.

$$
X_{t}=\sum_{i=1}^{p} \alpha_{i} X_{t-i}+\sum_{j=1}^{q} \beta_{j} \varepsilon_{t-j}+\varepsilon_{t} .
$$

The parameters $\alpha_{i}$ describe the impact of the values $X_{t-i}$ at the actual value $X_{t}$ for all $i=1, \ldots, p$. The parameters $\beta_{j}$ define the weights of the error terms (innovations) $\varepsilon_{j}$ within the moving average component. For an extensive discussion and applications concerning ARMA models for electricity prices see [16].

Typically ARMA models are used in time series analysis to account for linear serial dependence. They provide the possibility to condition the mean of the process on past realizations which has often produced acceptably accurate predictions of time series in the short term. However, the assumption of autoregressive model of conditional homoscedasticity is too constricting, as electricity prices usually display volatility clusters or spikes. That is, the variance is not constant within all parts of the time series, but there are rather phases of higher and lower volatility. During the phases of high volatility, markets are often nervous and electricity prices jump and remain longer in the jump-regime, implying a higher conditional probability for high price changes, when such price movements have already occurred in the recent past (see [16]).

Within the GARCH approach the assumption of homoscedasticity dropped for a heteroscedastic variance, meaning that the variance is not constant within all parts of the time series. The GARCH(p,q) process accordingly to [4] and [10] reads: 


$$
\sigma_{t}^{2}=\phi_{0}+\sum_{z=1}^{m} \phi_{1 z} \sigma_{t-z}^{2}+\sum_{y=1}^{n} \phi_{2 y} \varepsilon_{t-y}^{2}
$$

The time-variant variance $\sigma_{t}^{2}$ is driven by a constant component $\phi_{0}$, the autoregressive part of order $m$ and a moving average part of order $n$. The variance at any time $t$ must be positive and in consequence the parameters $\phi_{0}, \phi_{1 z}$ and $\phi_{2 y}$ can take only positive values, or equal zero at any time.

Researchers applying GARCH processes to model electricity prices assume that these can handle the heteroscedasticity caused by jumps in the case of electricity prices. However, beside seasonality and volatility clustering an important characteristic to be considered are the large number of extreme price changes. The spiking behavior is often described in the literature by regime switching models ([16], [3]) which allow electricity prices to switch between the normal or "base" regime and the "jump regime". Another modeling approach apply extreme value theory (EVT) to model the extreme tails of the electricity prices ([7]). We offer such an example in the next section.

\section{Extreme value theory for tail-quantile estimates}

One method to deal with extremely large price movements is to delimitate the extreme tail of the distribution and to model it independently with the POT (Peaks over the threshold) method, applying EVT (Extreme Value Theory) and GPD (Generalized Pareto Distribution). We show the comparison of the modeling performance of an AR-GARCH model with normal or $t$-distributed innovations against the conditional GDP and POT method applied to tail quantiles for EEX Phelix hourly electricity prices, for the sample period August 2008-August 2011. Compared to typical financial assets like stocks and bonds, the magnitude of the price changes in the EEX Phelix is extreme. As seen in figure 9, the exchange electricity prices have some hours go as high as $496.26 \mathrm{EUR} / \mathrm{MWh}$ and some hours go as low as -500 EUR/MWh.

Since the price changes are so extreme in some hours and we focus on the extreme quantiles of the distribution, we have chosen to use simple net returns $r_{t}=\left(P_{t}-P_{t-1}\right) / P_{t-1}$ instead of logarithmic returns. A similar methodology was suggested by [7]. The drawback of working with simple net returns if one is interested in large price drops is that there is a lower bound of $-100 \%$. We therefore focus in this study on the positive tail.

We model the seasonality of electricity prices in the mean equation of the ARGARCH specification. We therefore include AR(1) and AR(24) terms in the model to account for the daily electricity prices seasonality. We end up with the following specification: 


$$
\begin{aligned}
& r_{t}=a_{0}+a_{1} r_{t-1}+a_{2} r_{t-24}+\varepsilon_{t} \\
& \sigma_{t}^{2}=\phi_{0}+\phi_{1} \varepsilon_{t-1}^{2}+\phi_{2} \sigma_{t-1}^{2}
\end{aligned}
$$

where $\sigma_{t}^{2}$ is the conditional variance of $\varepsilon_{t}$. $\varepsilon_{t}$ is equal to $\sigma_{t} \eta_{t}$ with $\eta_{t} N(0,1)$ or Student's $t$-distributed i.i.d. innovations (scaled to have variance one) with mean 0 , variance 1 and degree of freedom $v$. The reason for including the $t$-distribution is that empirical evidence strongly rejects the idea that electricity price changes are normally distributed (see [7]).

We fit both versions of the AR-GARCH model to data by maximizing the likelihood function. Results are available in table 3. Likelihood ratio test results show a better performance of the AR-GARCH model with $t$-innovations against the version with normal innovations. For both AR-GARCH models (with Gaussian and $t$ distributed errors) we get significant parameter estimates and " $a$ "-parameters that are positive; a fairly high $R^{2}$ shows that the model explains successfully the data. The sum of the " $a$ "-parameters is not significantly lower than one, however, an infinite unconditional variance cannot be rejected for any of the two models. This is not surprising considering the extremely fat-tailed data in our study and it is further supported by the large positive tail-index estimate for the residual series and, particularly, for the original return series.

To check how much of the autocorrelation has been removed by our AR-GARCH volatility model, we look at the autocorrelation function of the standardized residuals $z_{t}$. To see how much of the heteroscedasticity has been removed by the GARCH model, we will analyze the filtered residuals $\varepsilon_{t}$. Figure 10 shows that an independent and identical distribution (i.i.d.) series is now approximately given for the standardized residuals of EEX Phelix price returns. The standardized residuals are meant to constitute the i.i.d. series with zero mean and unit variance that is used to estimate the tails of the sample cumulative distribution function with EVT. In figure 11 one can clearly see the heteroscedasticity in the filtered residuals. Most of the heteroscedasticity of the original data is reflected by our GARCH variance model.

We further investigate the tails of the price change distribution and estimate tail quantiles $\alpha_{t, p}$. We take standard quantiles of the normal distribution or of the $t$ distribution, multiply them with estimates of $\sigma_{t}$ and derive conditional tail quantiles $\alpha_{t, p}$ calling the mean equation of our AR-GARCH model:

$$
\alpha_{t, p}=a_{0}+a_{1} r_{t-1}+a_{2} r_{t-24}+\sigma_{t} \alpha_{p}
$$

We further model extreme tail quantiles with EVT. That is, we focus on the observations in the residuals $\eta_{t}$ from the AR-GARCH model with normal innovations applying the POT method, following the procedure in [7]. We thus collect observations in the residual series that exceed a certain high threshold $u$ (see [9], as cited by [7]). The excess distribution $F_{u}(y)$ is given by:

$$
F_{u}(y)=P(R-u \leq y \mid R>u)=\frac{\left(F_{R}(u+y)-F_{R}(u)\right)}{1-F_{R}(u)}, \quad 0 \leq y \leq R_{F}-u
$$


Table 3 AR-GARCH parameters. Own calculations

\begin{tabular}{lcccc}
\hline \hline AR-GARCH parameters & \multicolumn{2}{c}{ Normal } & \multicolumn{2}{c}{ Student's t } \\
& Coeff. & Std. errors & Coeff. & Std. errors \\
\hline$a_{0} * 10^{2}$ & 1.5 & $(0.00015)$ & 0.037 & $(0.00008)$ \\
$a_{1}$ & 0.0110 & $(0.0023)$ & -0.0012 & $(0.0004)$ \\
$a_{2}$ & 0.8640 & $(0.0016)$ & 0.9817 & $(0.0004)$ \\
$\phi_{0} * 10^{4}$ & 5.1602 & $(0.000005)$ & 0.0416 & $(0.000007)$ \\
$\phi_{1}$ & 0.5788 & $(0.0048)$ & 0.4930 & $(0.0036)$ \\
$\phi_{2}$ & 0.4212 & $(0.0022)$ & 0.5068 & $(0.0036)$ \\
$v$ & & & & 2.5407 \\
\hline Likelihood & $35 ' 100$ & & $54^{\prime} 670$ & \\
$R^{2}$ & 0.853 & & & \\
DW stat. & 1.856 & & & \\
\hline \hline
\end{tabular}

where $y$ is the excesses over $u$, and $R_{F}$ is the right endpoint of $F_{R}$, which is the assumed distribution of the residuals $\eta_{t}$. For a high enough threshold $u$, [1] and [23] show that for a large class of distributions $F_{R}$ the excess distribution $F_{u}(y)$ can be approximated by the so called Generalized Pareto Distribution (GPD):

$$
\begin{aligned}
& G_{\xi, \alpha}(y)=\left[1-\left(1+\frac{\xi}{\alpha} y\right)\right]^{-1 / \xi}, \quad \text { if } \quad \xi \neq 0, \\
& G_{\xi, \alpha}(y)=1-e^{-y / \alpha}, \quad \text { if } \quad \xi=0 .
\end{aligned}
$$

for $0 \leq y \leq R_{F}-u$. $\xi$ is the tail index and $\alpha>0$ is just a scaling parameter. The parameters are determined by fitting the GDP to the actual data and by estimating the parameters with the maximum likelihood method. In general, the threshold $u$ is chosen within reasonable limits of $5-13 \%$ of the data. We look at the most extreme $10 \%$ upper tail of the standardized residuals and fit the GPD to the upper tail excesses over the threshold. Thus, our upper tail "starts" at 0.9339. The maximum likelihood estimators for the generalized Pareto distribution are given in table 4. The model fit for the upper tail of residuals is shown in figure 4 .

Table 4 Maximum likelihood estimators for the generalized Pareto distribution parameters

\begin{tabular}{cccc}
\hline \hline \multicolumn{2}{c}{ Lower Tails } & \multicolumn{2}{c}{ Upper Tails } \\
\hline$\xi$ & $\alpha$ & $\xi$ & $\alpha$ \\
0.5913 & 1.7230 & 0.6909 & 1.8377 \\
\hline \hline
\end{tabular}

Accordingly to [7], the unconditional EVT tail quantiles $\alpha_{p}$ of the residual distribution with certain probabilities $p$ is given by:

$$
\alpha_{p}=u+\frac{\alpha}{\xi}\left(\left(\frac{n}{N_{u}} p\right)^{-\xi}-1\right)
$$




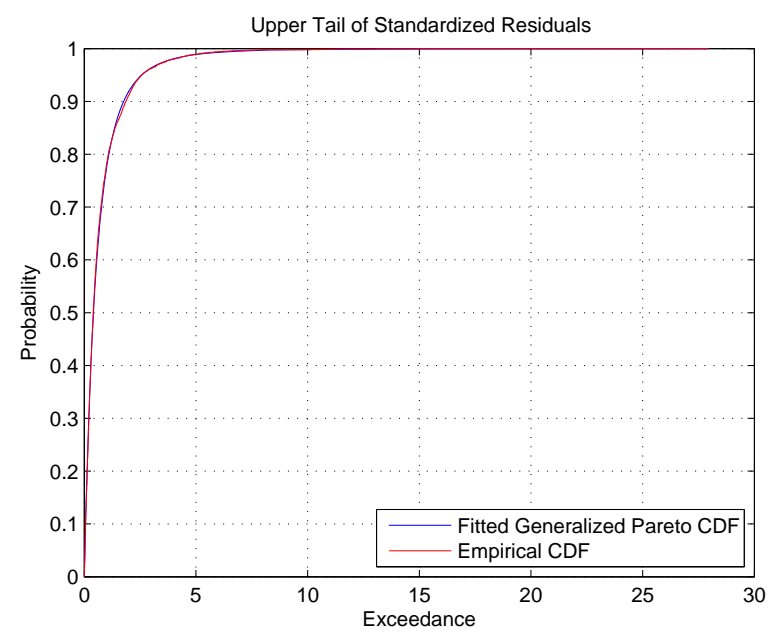

Fig. 4 Generalized Pareto upper tail of residuals

where $n$ is the total number of observations and $N_{u}$ is the number of observations above the threshold $u$. Conditional GPD tail quantiles are further obtained by calculating equation (16) for $\alpha_{p}$.

By counting the number of returns that are larger than the estimated tail quantile for each model version against the theoretical number of exceedences, we get a number that represents the accuracy of these estimates. Our estimated sample is extended over a period of 27414 hours. In this case, we compute the theoretical number of exceedences of a $99 \%$ tail quantile over a time period of 27414 hours as: $0.01 \cdot 27414=274.14$.

In the similar way we compute the theoretical exceedences of $95 \%, 99.5 \%$, $99.9 \%, 99.95 \%$ and $99.99 \%$ (extreme quantiles).

In table 5 we present the number of exceedences of the AR-GARCH-based tail quantiles for different tail probabilities. If a particular method to calculate marginal quantiles works well, then the empirically observed number of exceedences should be close to the theoretically expected. By comparing the AR-GARCH with normal or $t$-innovations we conclude that the shape of the conditional error term distribution has an important impact on the tail-quantile estimates. Neither the conditional normal distribution nor the conditional $t$-distribution captures the behavior of the positive tail in a more realistic way. We observe that the performance of the normal model gets worse, the higher the probability for the extreme tail is chosen, while for the $t$-distributed model we observe a slightly better fit. Similar results where found by [7] in an analysis of Nord Pool electricity prices. The interpretation is that AR-GARCH models describe the entire distribution of returns, not only the most extreme ones. Therefore they are not very successful in capturing extremely large price movements in electricity prices. Similar discussions can be found in [16]. 
The number of exceedences obtained with the POT method (conditional GPD) are close to the empirical ones for all quantiles. The unconditional EVT-based risk estimator has the advantage of treating the tails separately and thus more efficiently. Extending their analysis with out-of-sample tests and price forecasts, [7] conclude a high performance of the POT method in fitting extreme tails of hourly electricity prices. This is of significant importance for risk managers in determining accurate portfolio "value-at-risk". Additionally, a realistic approach for modeling the tails helps power portfolio managers for the estimation of worst-case scenarios in the context of stress testing.

Table 5 In-sample evaluation of estimated tail quantiles at different probabilities (number of exceedences)

\begin{tabular}{lrrrr}
\hline \hline Probability & Expected & AR-GARCH & AR-GARCH-t & Conditional GPD \\
0.95 & 1371 & 1086 & 1232 & 1577 \\
0.99 & 274 & 603 & 509 & 300 \\
0.995 & 137 & 464 & 370 & 156 \\
0.999 & 27 & 291 & 167 & 24 \\
0.9995 & 14 & 253 & 116 & 13 \\
0.9999 & 3 & 193 & 38 & 1 \\
\hline \hline
\end{tabular}

\section{Summary}

In this paper we present different modeling approaches for electricity prices. An overview of the main deseasonalization techniques is given. The price characteristics reflected by one or another model are discussed. Additionally the performance of some popular financial models is assessed in parallel: Brownian motions, Ornstein-Uhlenbeck processes and the Pilipovic model. We reveal the importance of modeling the mean-reversion and show that it makes sense to separate the shortfrom the long-term dynamics of electricity prices.

We further assess the performance of Extreme Value Theory (EVT) to model extremely large price movements. This procedure gives much more realistic tail estimates than the classical time-series models. Realistic tail quantiles estimates for the electricity prices are of high interest for both risk managers and portfolio managers in the high volatile electricity markets.

In this respect, the choice of the modeling approach depends on the different research questions or planning tasks. 


\section{Appendix}
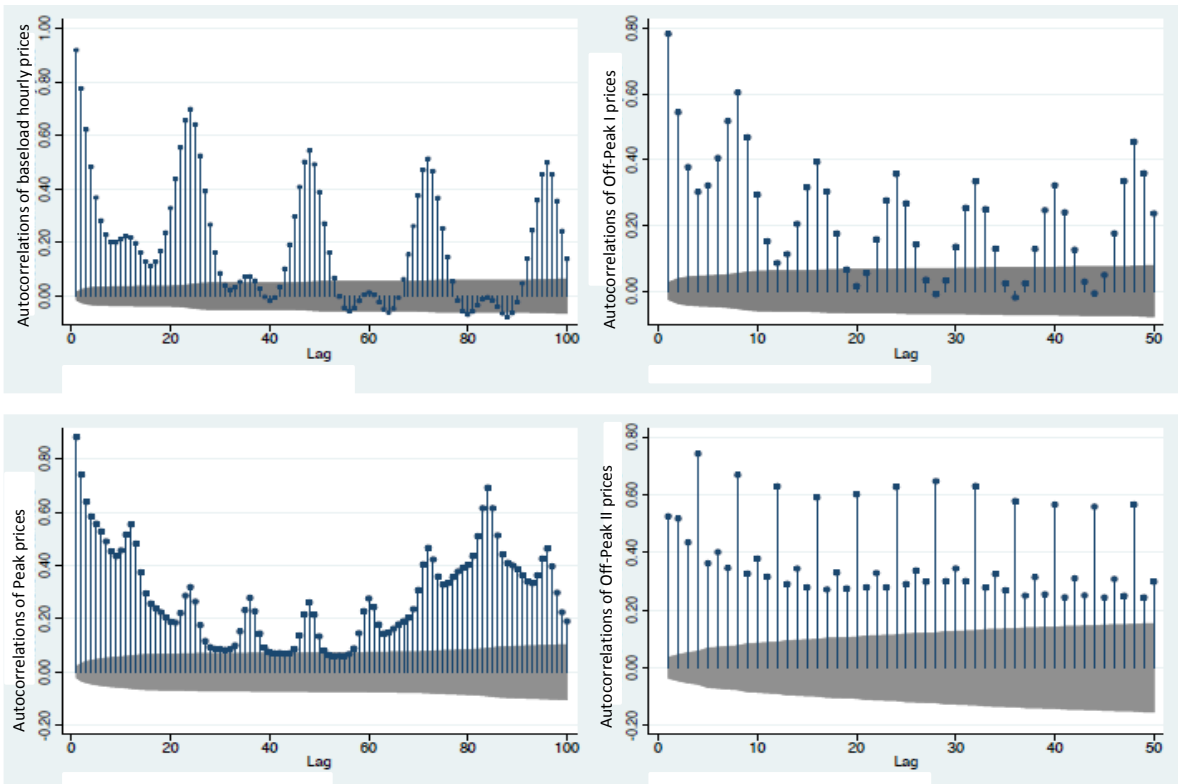

Fig. 5 Autocorrelations of day-ahead baseload, off-peak I, peak and off-peak II hourly prices (source [11], pp. 35) 


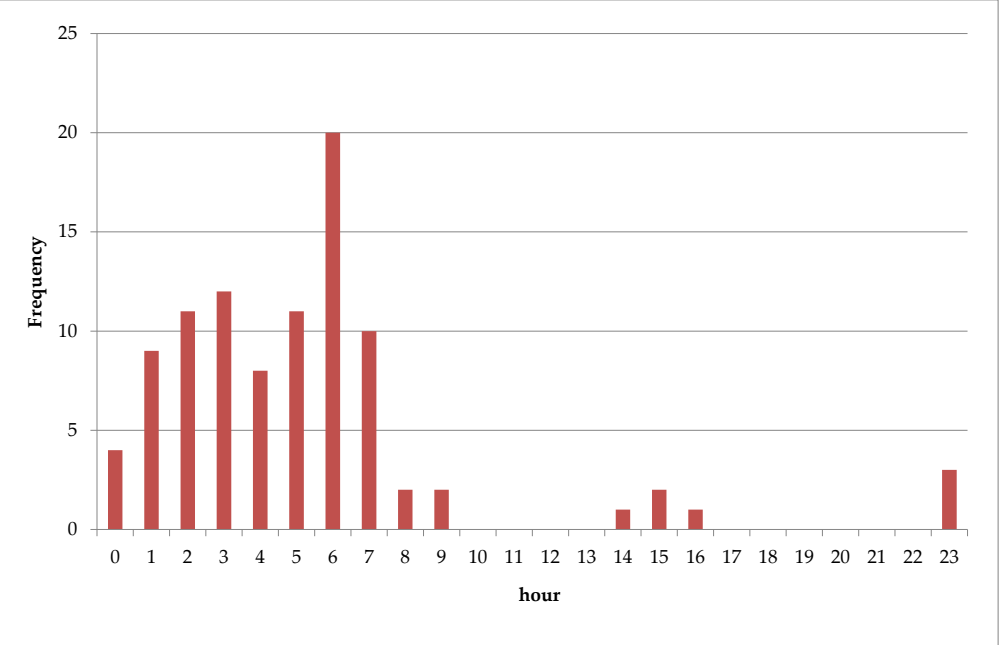

Fig. 6 Occurrence of negative prices Sept. 2008-Dec. 2011 on different hours

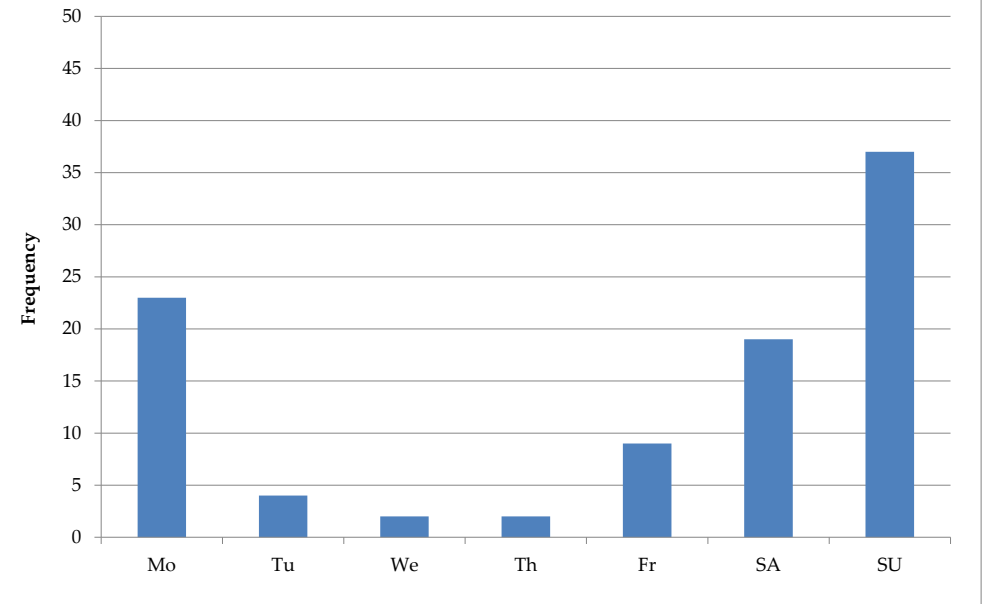

Fig. 7 Occurrence of negative prices Sept. 2008-Dec. 2011 on different days 


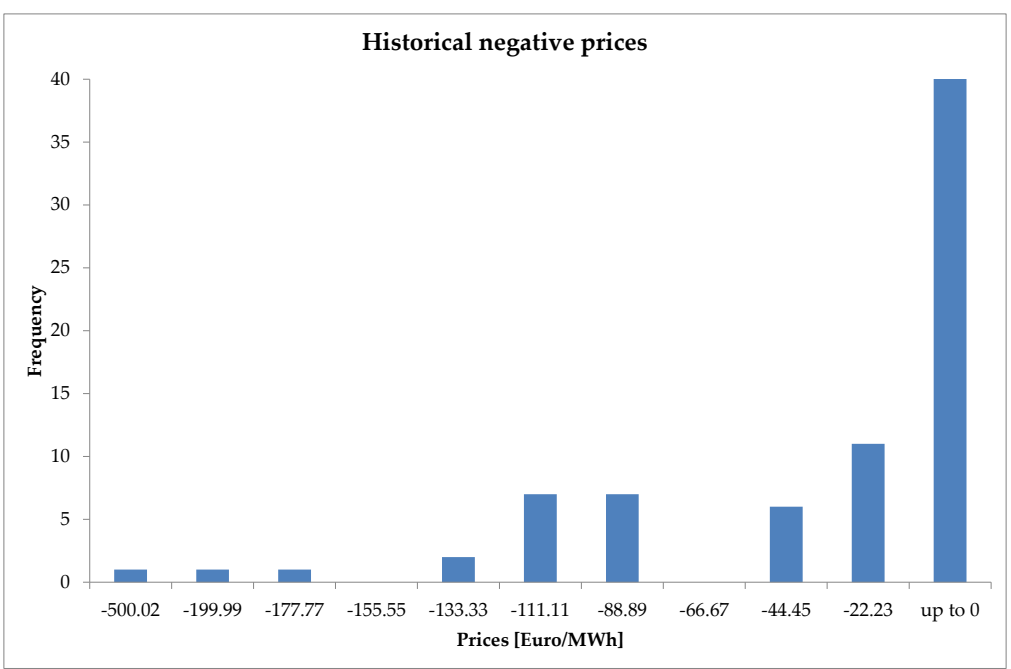

Fig. 8 Histogram of historical negative prices at EEX

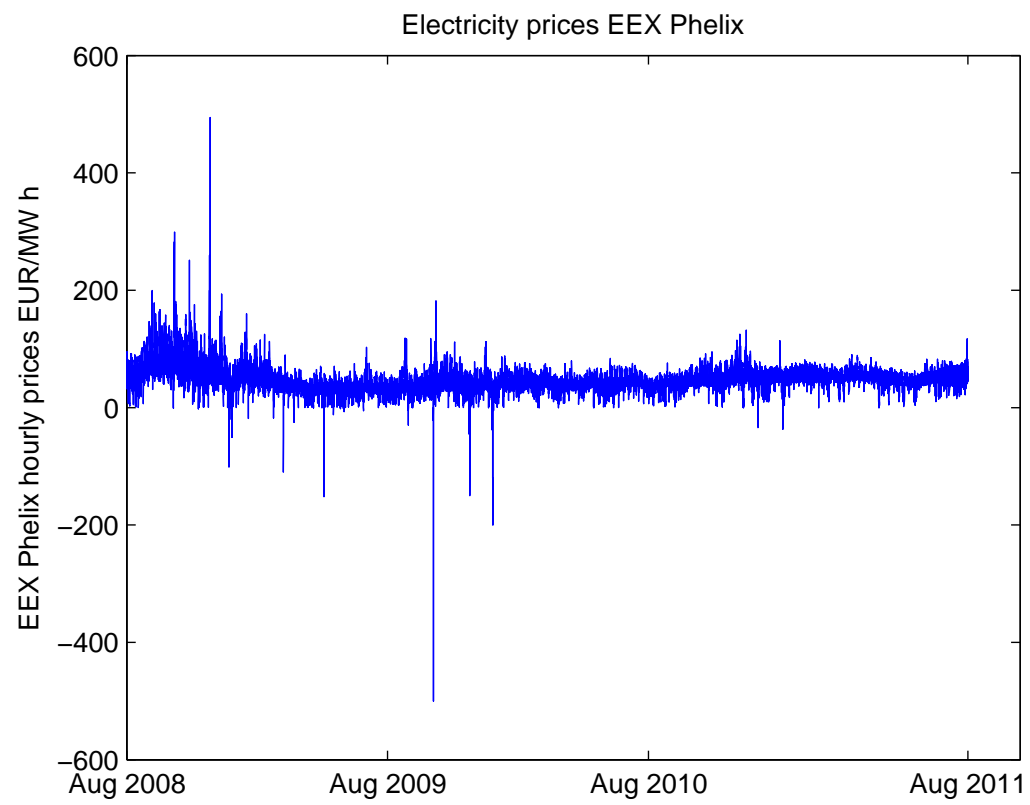

Fig. 9 EEX hourly prices 

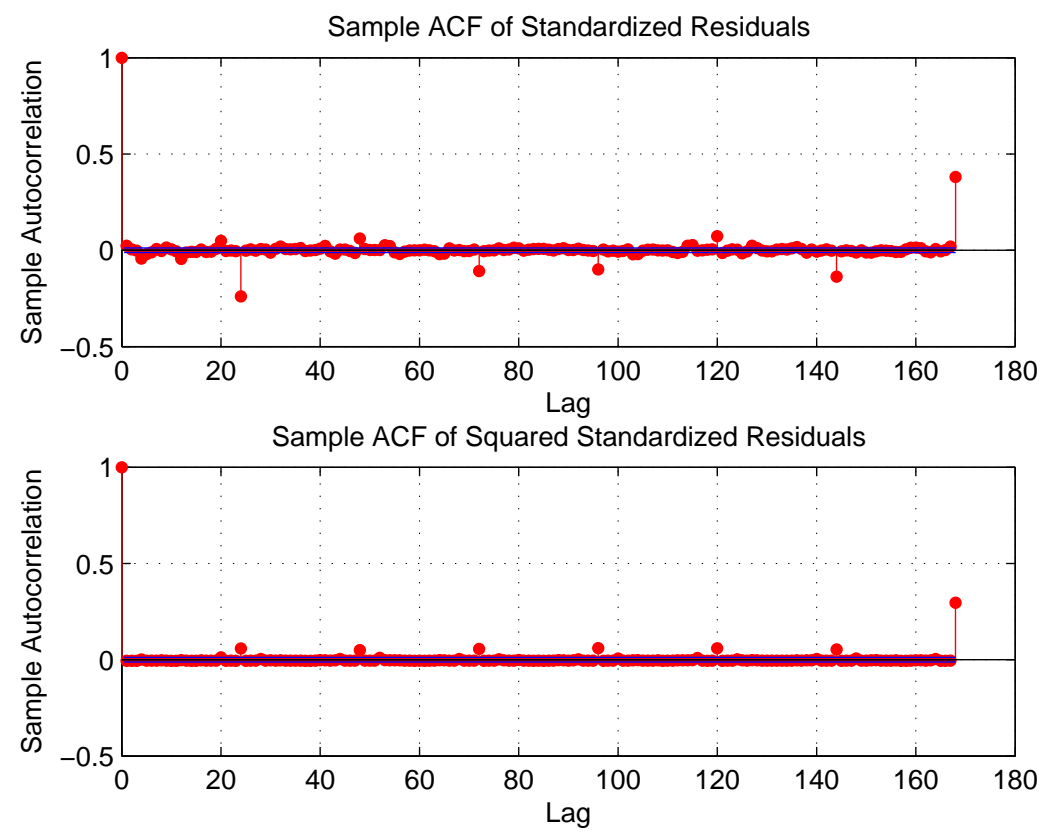

Fig. 10 Autocorrelation function of standardized residual
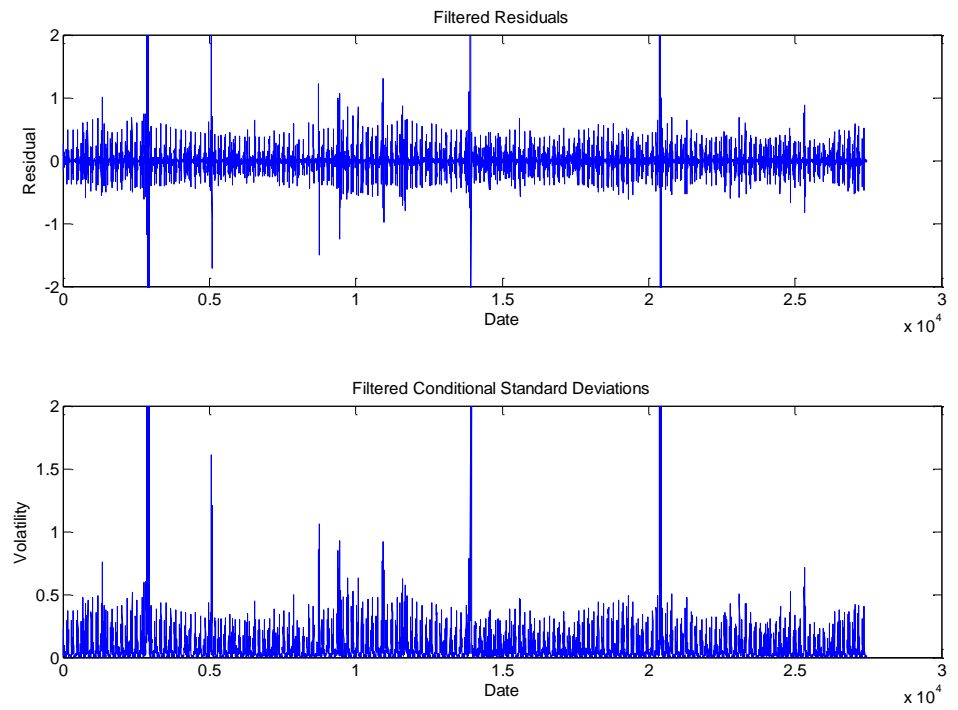

Fig. 11 Filtered residuals and filtered conditional standard deviation 


\section{References}

1. Balkema, A., de Haan, L.: Residual life time at great age, Annals of Probability 2, 792-804, (1974)

2. Bierbrauer, M., Menn, C., Rachev, S.T., Trück, S.: Spot and derivative pricing in the EEX power market, Journal of Banking and Finance 31, 3462-3485, (2007)

3. Blöchlinger, L.: Power prices - A regime-switching Spot/Forward price model with Kim filter estimation. Dissertation, University of St. Gallen, 3442, (2008)

4. Bollerslev, T.: Generalized autoregressive conditional heteroschedasticity, J. Econ 31(3), 307327, (1986)

5. Borchert, J., Schemm, R., Korth, S.: Stromhandel. Institutionen, Marktmodelle, Pricing und Risikomanagement, Stuttgart: Schäffer-Poeschel, (2006)

6. Burger, M., Klar, B., Müller, A., Schindlmayr, G.: A spot market model for pricing derivatives in electricity markets, Journal of Quantitative Finance 4(1), 109-122, (2003)

7. Byström, N.E.: Extreme value theory and extremely large electricity price changes, International Review of Economics \& Finance 14, 41-55, (2005)

8. Cartea, A. \& Figueroa, M.G.: Pricing electricity markets: a mean-reverting jump diffusion model with seasonality, Applied Mathematical Finance 12(4), 313-335, (2005)

9. Embrechts, P, Kluppelberg, C., Mikosh, T.: Modeling extremal events, Berlin: Springer, (1997)

10. Engle, R.F.: Autoregressive conditional heteroschedasticity with estimates of the variance of United Kingdom Inflation, Econometrica 50(4), 987-1007, (1982)

11. Geninasca, D.: Wind power feed-in-effects on prices, Master thesis, University of St. Gallen, (2012)

12. Geman, H., Roncoroni, A.: Understanding the fine structure of electricity prices, Journal of Business 79(3), 1225-1261, (2006)

13. Gibson, R., Schwartz, E.S.: Stochastic convenience yield and the pricing of oil contingent claims, Journal of Finance 45(3), 959-976, (1990)

14. Hoobs, B.F.: Linear complementarity models of Nash-Cournot competition in bilateral and POOLCO power markets, IEEE transactions on pwer systems 16(2), 194-202, (2001)

15. Janczura, J., Weron, R.: An empirical comparison of alternate regime-switching models for electricity spot prices, Energy Economics 32, 1059-1073, (2010a)

16. Keles, D., Genoese, M., Möst, D., Fichtner, W.: Comparison of extended mean-reversion and time series models for electricity spot price simulation considering negative prices, Energy Economics 34, 1012-1032, (2011)

17. Knittel, C.R., Roberts, M.R.: An empirical examination of deregulated electricity prices, POWER working paper, University of California, (2001)

18. Lise, W., Lindeerhof, V., Kuik, O., Kemfert, C., Ostling, R., Heinzow, T.: A game theoretic model of the Northwestern European electricity market - market power and the environment, Energy Policy 34, 2123-2136, (2006)

19. Lucia, J.J., Schwartz, E.S.: Electricity prices and power derivatives: evidence from the nordic power exchange, Review of Derivatives Research 5(1), 5-50, (2002)

20. Manoliu, M., Tompaidis, S.: Energy futures prices: term structure models with Kalman filter estimation, Applied Mathematical Finance 9(1), 21-43, (2002)

21. Nicolosi, M.: Wind power integration and power system flexibility - an empirical analysis of extreme events in Germany under the new negative price regime, Energy Policy 38, 7257$7268,(2010)$

22. Nowicka-Zagrajek, J., Weron, R.: Modeling electricity loads in California: ARMA models with hyperbolic noise, HSC Research Report 02/02, Wroclaw University of Technology, Wroclaw (Poland), (2002)

23. Pickands, J.: Statistical inference using extreme order statistics, Annals of Statistics 3, 119131, (1975)

24. Pilipovic, D.: Energy Risk: Valuing and Managing Energy Derivatives, New York: McGrawHill, (1997) 
25. Pilipovic, D.: Energy Risk: Valuing and Managing Energy Derivatives, 2nd edition, New York: McGraw-Hill, (2007)

26. Schwartz, E., Smith, J.: Short-term variations and long-term dynamics in commodity prices, Management Science 46(7), 893-911, (2000)

27. Simonsen, I., Weron, R., Mo, B.: Structure and stylized facts of a deregulated power market, Working paper (2004) http://mpra.ub.uni-muenchen.de/1443

28. Swider, D.J., Weber, C.: Extended ARMA models for estimating price developments on dayahead electricity markets, Electric Power Systems Research 77, 583-593, (2007)

29. Viehmann, J.: Risk premiums in the German day-ahead Electricity Market, Energy Policy 39, 386-394, (2011)

30. Weber, C.: Uncertainty in the electric power industry, Springer, New York, (2005)

31. Weron, R.: Modeling and forecasting electricity loads and prices: a statistical approach, Chichester: Wiley, (2006)

32. Weron, R., Bierbauer, M., Trück, S.: Modeling electricity prices: jump diffusion and regime switching, Physica A 336, 39-48, (2004) 


\section{Index}

ARMA process, 2, 13

autocorrelation, 3, 9, 13, 15, 19, 22

Brownian motion, 2, 10, 11, 13, 18

deseasonalization, 1, 2, 5, 7, 18

electricity prices, $1-18$

Extreme Value Theory, 1, 2, 14, 15, 18
GARCH process, 2, 13-15, 17

mean-reversion, $3,4,10,11,18$

negative prices, $1-3,20$

Ornstein-Uhlenbeck, 2, 10, 11, 13, 18

Pareto distribution, 14, 16-18

Pilipovic model, 2, 11, 12, 18 